\title{
ARTICLE
}

\section{Psychiatric assessments on medical wards: a guide for general psychiatrists}

\author{
Tim Segal \& Gopinath Ranjith
}

\begin{abstract}
Tim Segal is a Year 4 Specialist Trainee (ST4) in general adult psychiatry at the South London and Maudsley NHS Foundation Trust. He has completed a 6-month placement in liaison psychiatry during core training and has a clinical interest in psychiatric illness in the medically unwell. Gopinath Ranjith is a consultant liaison psychiatrist with the South London and Maudsley NHS Foundation Trust based at St Thomas' Hospital, London. He is also a visiting lecturer at the Institute of Psychiatry, Psychology and Neuroscience at King's College London and lead for foundation training at Maudsley Hospital. His clinical and research interests are in mood disorders in the medically ill, self-harm and suicidal behaviour, and process and outcome measures in consultation-liaison services. Correspondence Dr Gopinath Ranjith, South London and Maudsley NHS Foundation Trust, Scutari Clinic, St Thomas' Hospital, Westminster Bridge Road, London SE1 7EH, UK. Email: gopinath.ranjith@slam.nhs.uk
\end{abstract}

\section{SUMMARY}

Psychiatric assessments on medical wards of a general hospital are complicated by various factors, including the lack of privacy, medical illness-related factors such as cognitive deficits and communication barriers, and the vagueness of the referral question asked. In this article, we try to guide general psychiatrists who do not routinely carry out such assessments through practical steps from receiving the referral to signing off the case. The key differences in the process and content of psychiatric assessments are discussed, as are diagnostic dilemmas. Subtle aspects of communicating the outcome of the assessments to patients and referrers are emphasised with specific guidance on writing consultation notes. We conclude with a discussion of the principles of biopsychosocial management and judging the outcomes of consultations on medical wards.

\section{LEARNING OUTCOMES}

- Gain an appreciation of the differences between a standard psychiatric assessment and an assessment of a patient in a general hospital inpatient setting

- Learn about dealing with a referral from a medical ward, from receiving the referral to formulating a management plan

- Learn about communicating the outcome of the assessment to the referrer and judging the success of the consultation

\section{DECLARATION OF INTEREST}

None

Psychiatrists are experienced in carrying out psychiatric assessments in a variety of settings, including inhospitable environments such as police stations, chaotic psychiatric wards and busy emergency departments. However, even for an experienced psychiatrist, performing a psychiatric assessment on a relatively settled medical or surgical ward may prove daunting. Problems the psychiatrist faces, particularly if their days of postgraduate training in the general hospital are but a distant memory, include the vagueness of the question being asked, the unfamiliar ward routines and hierarchies, the lack of privacy and the challenge associated with the medical illness itself, with patients often connected to medical devices and multiple lines. In this article we provide a practical guide to approaching an assessment on a medical ward from the moment a referral is received to when the psychiatrist signs off the case.

In many general hospitals assessments are carried out by liaison psychiatrists or other members of the liaison psychiatry team such as psychiatric liaison nurses. But in parts of the country liaison psychiatry services remain patchy, with community mental health services providing 'in-reach' services to patients from their catchment area. Even where there are liaison psychiatry services, they are often available only in office hours, leaving out-of-hours cover of the general hospital the responsibility of junior trainees supervised by general psychiatrists. Given the move towards 24/7 working and consultant-delivered services in the National Health Service (NHS), it is probable that consultant general psychiatrists will become more involved in assessments of patients in general hospitals, making this article especially relevant.

\section{The referral}

\section{Receiving the referral}

For a standard psychiatric assessment the reason for referral is usually pretty clear from the general practitioner's (GP's) letter, but this is not always the case for referrals from medical wards. The referral is usually made by a junior member of the medical or surgical team, who is carrying out tasks from a busy medical ward round and may not know the patient well. The mode of referral varies between hospitals, from a telephone call to a referral letter or, increasingly commonly, a generic referral form often completed on an electronic system. A more formal referral system is helpful as it ensures that all the essential basic information is 
B0X 1 Common reasons for referrals from medical wards

- Assessment and management of psychiatric disorder

- Impact of pre-existing psychiatric symptoms on medical disorder

- Medically unexplained physical symptoms

- Advice regarding psychotropic medications

- Management of agitated or challenging behaviour

- Suicide risk assessment and management

- Second opinion on decision-making capacity

given and it focuses the attention of the referrer on the question being asked. The basic information required includes the patient's demographic details (name, date of birth, hospital number), location in the hospital, the patient's primary diagnosis and reason for admission, the psychiatric concerns and reason for referral, the urgency of the assessment and the referrer's details. Common reasons for referral can be seen in Box 1.

\section{Clarifying the referral}

Sometimes the reason for referral and the nature of the assessment required are unclear (House 2012). It is therefore often useful to contact the referrer to get further information. In doing this you can get a better idea about the patient's medical and psychiatric condition and the urgency of the referral, so that a timely response can be agreed. You can also identify exactly what question the referrer wants answered. Practical issues can be broached, such as when the patient will be available, where the assessment can take place and whether there are challenges to communication such as a language barrier, tracheostomy or other medical devices. It is also important to clarify whether the referral has been discussed with the patient and whether the patient has consented to being seen by psychiatric services. Explaining to the patient that a psychiatrist is coming to see them prevents an awkward discussion on arrival with an unprepared patient who may feel offended that a psychiatrist has been called. The patient has the right to refuse psychiatric assessment, and this should be respected if possible. In general, the only reason for carrying out a psychiatric assessment of a non-consenting patient would be if there are initial grounds for believing that an assessment under mental health legislation may be indicated.

Even when the referral question has been clearly articulated, the underlying motive for requesting a psychiatric assessment may not always be the reason written in the referral form (Querques
2010). It is therefore important to be able to read between the lines of a referral (Box 2). For example, a referral for medically unexplained symptoms may belie frustration with a patient who is being repeatedly admitted to hospital. A referral for the management of agitation may involve a patient who is aggressive because his demands are not being met. Often it will become apparent on further discussion that it is the treating team's frustration with the patient that has resulted in the referral, rather than a psychiatric problem in the patient. Determining underlying reasons before starting the assessment will help guide your interview and, ultimately, your management plan.

\section{Appropriateness of the referral}

The final aspect of dealing with referrals is to decide whether to accept the referral. At one extreme is a general hospital psychiatric service 'that never says "No"' (Smith 1998); at the other is a strict screening system for referrals, with a significant number rejected as subthreshold. In our opinion it is best to chart a midway course. Before an assessment, it is certainly reasonable to ask for further investigation to rule out medical causes, or to ask the referrer to talk to the patient to get a better understanding of the psychiatric concerns. If it is clear from talking to the referrer that the problems relate to transient emotional reactions, purely antisocial behaviour unrelated

BOX 2 Reading between the lines of a referral (a fictitious example)

A 35-year-old woman admitted 2 weeks ago with abdominal pain received multiple investigations that showed mild gastritis, nil other significant. Increasing anxiety interfering with management, complex family dynamics. Nil psychiatric history. Please review for diagnosis/management of anxiety.

On further discussion with the referrer, it becomes clear that what is described as anxiety actually refers to the patient becoming increasingly demanding on the ward. She is requesting pain relief frequently and becoming verbally abusive when this is not given. Her family are often on the ward in large numbers, sometimes outside of visiting hours, and make regular threats of complaints about what they perceive as staff negligence. This is causing a significant strain on nursing and medical staff in terms of both time and morale. Armed with this information, the psychiatrist was aware that what was needed was a systemic formulation and management plan involving the patient, family and ward staff, rather than a straightforward assessment of mood and recommendation of medication and/or psychological therapy. 
to mental disorders or questions better directed at other professionals, such as social workers or lawyers, it is appropriate to reject the referral. However, caution should be taken with rejecting referrals without an assessment, as the referrer is often not a mental health expert and may have identified a problem but failed to articulate it well in the referral letter. As a general rule it is better to accept referrals unless there are clear reasons not to, as it is often difficult to make judgements about appropriateness without having at least briefly seen the patient.

\section{Pre-assessment preparation}

There are two aspects of preparing for a liaison psychiatry assessment: the practical preparation and the acquisition of background information.

\section{Practical preparation}

First, you must ensure that there is a suitable time and location for the assessment to take place. It is usually worth contacting the ward to arrange a time that does not clash with activity on the ward such as ward rounds, assessments by other specialties and allied health professionals, protected mealtimes, planned visitors and other potential interruptions. It is also worth locating an appropriate place to perform the assessment. The issues discussed in a psychiatric assessment are often more personal than in a general medical history and people may be less forthcoming if the assessment is not conducted in a private area. In spite of the illusion of privacy provided by curtains around a patient's bed, they are not soundproof. A quiet side-room may well create an environment more conducive to obtaining a thorough and open assessment, but the consulting psychiatrist has to be flexible enough to proceed with the assessment in any environment, as in the case of a patient on an intensive care unit.

\section{Obtaining background information}

Second, you must gain the appropriate background information. It is essential to find out whether the patient has a history of psychiatric problems: this might involve searching local notes, contacting mental health services where the patient is from or talking to the patient's GP. Looking through psychiatric records and clinic letters for past mental state examination, cognitive assessment and use of psychotropic medications yields valuable information. Following this, it is important to understand the patient's current and past medical history by looking through their medical notes. This should include the patient's current diagnosis, investigations, ongoing medical plan, a comprehensive list of medications, the prognosis and the planned discharge date if appropriate (Lackamp 2010; Smith 2011). In our experience it is important to pay particular attention to entries made by nurses and allied health professionals (such as physiotherapists and dieticians), as they often provide better clues to behavioural and emotional issues. Finally, having gained this information you may have to do some background reading about the patient's medical illness. Many patients are experts in their medical condition and having a good understanding of the condition enhances your credibility when interviewing the patient. This level of preparation ensures that the assessment process goes as smoothly as possible.

\section{The assessment}

\section{Assessment process}

As well as the practicalities of the environment of a general hospital as described above, there are also the challenges posed by assessing patients with comorbid physical conditions. Often patients seen on a general ward are physically disabled or significantly unwell. First, you have to decide whether the patient is fit to be assessed: if they cannot maintain a clear conversation, assessment may be better left until they can. If this is not feasible, a compromise may be more appropriate: the assessment can be reduced in duration and split over several visits. There may be permanent limitations to communication, such as longstanding intellectual disability, acquired language disorder due to stroke, tracheostomy or simply poor English language skills. These will have to be dealt with on an individual basis, and may involve the use of interpreters, speech boards, written interviews or, at times, sheer patience and perseverance.

\section{Content of the assessment}

The structure of the assessment on a medical ward does not differ very much from a standard psychiatric assessment, with a few exceptions. Even if the psychiatric referral was discussed with the patient beforehand and the patient had consented, it cannot be assumed that they have fully accepted the idea. Thus, except in cases where the patient requested the referral, it is advisable to ask the patient about their views on the referral and to normalise the consultation in the context of a stressful hospital admission. It is also usually a good idea to spend the initial few minutes discussing the presenting physical illness. This not only establishes you as an integral part of the larger medical team, but can also elicit 
BOX 3 Relationship between physical and psychiatric illness

- Psychiatric presentations of medical disease

- Psychiatric complications of medical disease or treatment

- Psychological reactions to medical disease

- Medical presentations of psychiatric disorders

- Medical complications of psychiatric disorders or treatments

- Comorbid medical and psychiatric conditions

(Adapted from Lipowski 1967)

concerns or complaints the patient has about their illness, its management and the treating professionals. During the assessment you should try to establish the relationship between the physical and psychiatric problems: the schema described by Lipowski (1967) is a practical way of thinking about this relationship (a modified version is shown in Box 3). It is also important to elicit the patient's explanatory model, as this will be crucial in developing a shared formulation and management plan. A cognitive screening test is necessary in most cases and an extended bedside cognitive examination is indicated wherever cognitive deficits are suspected.

\section{Diagnostic issues}

\section{Diagnostic challenges}

Issues related to psychiatric diagnosis and classification in the medically ill are beyond the scope of this article and have been discussed in detail elsewhere (Ranjith 2012). In addition to giving a diagnosis according to an accepted classificatory system, it is also important to formulate the patient's psychiatric problems in language that can be understood by nonpsychiatrists. Common diagnostic issues relevant to psychiatric assessments on general ward include the differentiation of 'organic' and 'functional' aetiologies of psychiatric symptoms and syndromes, medically unexplained physical symptoms and the differential diagnoses of low mood in the medically ill. To illustrate diagnostic dilemmas we have chosen to discuss the topic of emotional reactions in the medically ill and we also draw attention to the phenomenon of diagnostic overshadowing.

\section{Diagnostic dilemmas: an example}

Deciding when emotional reactions to physical illness warrant a psychiatric diagnosis can be a challenge in itself. Differentiating between adjustment disorders, demoralisation and depressive illness in people who have chronic medical conditions is difficult, as the stressor is not a discrete life event but often a long-lasting medical illness (O'Keeffe 2007). One must appreciate that a normal response to illness and disability, particularly initially, is to feel low and upset, and certainly psychiatry as a profession should avoid medicalising the normal. As Kontos et al (2003) point out, sometimes it can be useful to document this: for example, 'This patient is certainly sad and suffering, but at this point there is no evidence for, or benefit in, pathologizing that suffering'. On the other hand, there is the risk of what Lyketsos \& Chisolm (2009) call the 'trap of meaning', whereby genuine depressive disorders are dismissed as understandable reactions to difficult life events such as serious medical illness. Although diagnostic criteria are undoubtedly useful, symptoms such as insomnia, weight loss and low energy levels are not always due to depression. It is also difficult to reliably assess features such as anhedonia in the hospital setting. Ultimately, the psychiatrist has to rely on clinical judgement, assessing features such as the pervasiveness and severity of the mood change, its distinct quality and impact on functioning (including, in the hospital, the ability to cooperate with medical treatment) to determine when the depression has taken on a life of its own and needs management.

\section{Diagnostic overshadowing}

When treating patients with mental illnesses in a general medical setting it is important to be aware of, and overcome, the challenge of diagnostic overshadowing. It is all too common that a patient's psychiatric symptoms on the ward are attributed to an existing mental illness, and they are not warranted the full range of investigations they would normally have. For example, disorientation and agitation in an older patient may be attributed to their underlying dementia, so the medical team fails to carry out a full delirium screen to look for treatable causes. Similarly, a pre-existing diagnosis of schizophrenia in the medical record may preclude a full work-up for stroke in a patient presenting with incoherent speech. Psychiatrists must take care to spot and challenge this misuse of Occam's razor (Kontos 2008). It should not be forgotten that patients with psychiatric illness are at significant risk of comorbid physical conditions in relation to medication use and lifestyle choices (Royal College of Psychiatrists 2009). Furthermore, some psychiatric patients have difficulty expressing their physical symptoms 
or are reluctant to seek help. Mental health professionals play an important role as advocates for these individuals to ensure that their needs are met.

\section{Management}

\section{Medical advice and medications}

Once assessment and diagnosis are completed, the focus turns to management. On a medical ward the psychiatrist is often acting in a consultative capacity rather than as the primary clinician. The process of management takes on a more advisory nature and decisions have to be discussed with the relevant clinical team. This advice can take several different forms, often depending on the nature of the referrer's question.

It is important for psychiatrists to keep their medical hat firmly on. This might mean making an unequivocal diagnosis of delirium and encouraging the physicians to search for an underlying cause, or suggesting further medical investigations, such as testing for antibodies for autoimmune encephalitis in a patient presenting with a first episode of atypical psychotic symptoms.

Given the unfamiliarity of most hospital physicians with psychotropic medications, it is not surprising that queries about them are often addressed to psychiatrists. Examples of typical questions include: the best antidepressant to be used following myocardial infarction, the best antipsychotic to be used in someone with a prolonged QTc interval, and whether a selective serotonin reuptake inhibitor antidepressant needs to be stopped in someone with a gastrointestinal bleed. Even when the referral query is not about psychotropic medications, they are often used in the management of psychiatric disorders in patients with organ dysfunction who are taking multiple co-prescribed drugs. It is not possible for psychiatrists to memorise every potential drugdisease or drug-drug interaction and it is important to have access to trusted sources of information, such as a mental health pharmacist or a medicines information service, or to have reference resources close at hand (Taylor 2012; Bazire 2014). There are also trustworthy websites to consult for drugdrug interactions. If you prescribe a psychotropic medication, it is essential to follow up the case, not only to monitor the patient's response to the treatment, but also to monitor for any side-effects.

\section{Psychosocial management}

It is rare that medications are the sole intervention and the management plan needs to incorporate psychosocial aspects as well. Although structured psychotherapy may not be possible at the medical bedside, addressing psychological problems in a supportive way should be part of the toolbox of every psychiatrist assessing patients on medical wards. A good example of this approach is the brief bedside psychotherapy for countering demoralisation described by Griffith \& Gaby (2005). Other psychological techniques, such as relaxation exercises, problem-solving and behavioural activation, are also feasible in a hospital context. There may be times when the intervention is addressed not at the patient but at the medical team, for example when the team is struggling with a 'difficult' patient who does not have a diagnosable psychiatric problem. It is not uncommon that the consulting psychiatrist becomes involved in disputes that arise over the care of patients. These might be between patients and/or family and medical professionals, between different professionals caring for the patient, or between the medical and psychiatric teams. In such situations it is best to take a systemic view, encouraging open communication between the parties involved and taking advice from senior members of the teams.

Psychiatrists often contribute to a patient's care by initiating referrals to appropriate agencies such as social workers, hospital chaplains and homelessness services. Even in cases where the primary problem is social, an offer from a psychiatrist to attend a multidisciplinary case conference and clarify psychiatric questions is usually well received. Psychiatric care plans should involve advice about safe and appropriate nursing, including behavioural contracts or constant nursing observation. When constant observation by a mental health nurse is recommended it is important to specify the reason for the recommendation and regularly review the level of observation.

\section{Discharge planning}

The next stage of management is often the process of discharge planning and deciding on the discharge destination. The initial question that needs to be answered is whether it is safe to send the patient home, with or without the involvement of home treatment or community mental health teams, or whether they require admission to a psychiatric unit. If a patient is being transferred to a psychiatric unit, it falls to the psychiatrist in the general hospital to make sure that the patient is 'medically cleared'. Medical clearance is a thorny issue that eludes definition; the general hospital psychiatrist is often in the tricky position of having to explain to the medical team the limitations of physical health monitoring 
and interventions on psychiatric wards, and also to detail to the psychiatric team all the medical investigations and treatments that have been carried out thus far. There are times when the most effective strategy is to let doctors from the medical and psychiatric teams speak to each other. When patients are transferred it is essential that copies of medical notes, medication charts and investigation reports are made available to the psychiatric ward team.

\section{Legal and ethical issues}

Psychiatrists providing consultations on medical wards need to ensure that patients are treated within the right legislative framework and they need to have a good understanding of the relevant legislation. In England and Wales, this is the Mental Capacity Act 2005 (Department for Constitutional Affairs 2007), the Mental Health Act 1983 and the Deprivation of Liberty Safeguards (Ministry of Justice 2008). In addition to knowledge about the use of these legal powers, they should also be familiar with the local protocols and service level agreements between the general hospital and the mental health trusts in order to ensure that their use remains valid. Details of the use of the Mental Health Act in the general hospital are beyond the scope of this article and the reader is advised to consult sources of information such as Zigmond (2014).

With regard to the assessment of capacity in cases of treatment refusal, to avoid fruitless arguments hospitals should have clear referral criteria regarding when psychiatrists should be involved in joint capacity assessments with the physician or surgeon proposing the treatment. It is also important that psychiatrists do not set themselves up as the legal and ethical experts for all difficult treatment decisions: the medical teams should be encouraged to consult hospital lawyers or clinical ethicists in such situations.

\section{Communication}

\section{Communication with the patient}

It is a truism to state that good communication skills are essential throughout medicine in general and psychiatry in particular. We discuss it separately here because of the subtle yet significant requirements of communication within a psychiatric assessment on a medical ward. As the environment at the bedside on a medical ward is different from that in an out-patient clinic, you may need to take a more active stance, offering to help patients with simple tasks such as getting them a drink or adjusting the bed so that they are comfortable. It is important to tell the patient what you understand of their medical illness, elicit their narrative of their illness and their concerns, and to show curiosity about non-illnessrelated aspects of their life, such as their personal interests, hobbies and achievements. This will give a better understanding of the person behind the illness. When interviewing patients demoralised by their medical illness, it is essential to adopt an interviewing style that avoids colluding with their hopelessness but at the same time eschews a false sense of cheerfulness. At the end of the interview it is essential to summarise the salient details, agree a shared formulation of the patient's problems, involve them in decision-making and make them aware of follow-up arrangements both while they remain in hospital and after they have been discharged.

For a more comprehensive discussion of bedside communication see the article by Yager (1989).

\section{Communication with the referrer}

The second element of communication relevant to an assessment on a medical ward is how information is conveyed back to the referrer. It is important to inform the referrer of your assessment directly, by telephone or in person, and to hand over any advice or plans. Before you leave the ward, it is good practice to speak to the nurse looking after the patient. It is essential to ensure that the documentation is clear in the patient's notes. This is particularly important when, as is the case in most hospitals in England, the psychiatric and medical teams belong to different organisations, each with their own electronic health record systems to which the others do not have ready access.

Alexander \& Bloch (2002) suggest a schema for a written report which is comprehensive and concentrates on elements specific to consultation on a medical ward. However, our recommendation, based on our experience of dealing with two sets of records, is to enter a short, pithy note into the medical notes and to make a comprehensive entry in the psychiatric record that is made available to the medical team if required or on request. The note, an example of which is shown in Box 4, consists of a brief summary, an impression and a management plan. It is likely that a busy physician will read little more than the last. Although it is important not to withhold significant information from the medical team, one should also remember that information that the patient gives in the more intimate setting of a psychiatric assessment may not necessarily be relevant to their ongoing physical care and therefore may not need to be recorded in the medical notes. 
BOX 4 Example consultation note on a fictitious patient

Ms P, a 36-year-old teacher with Crohn's disease and no history of mental illness, was admitted to hospital 3 months ago with acute abdominal pain and underwent a hemicolectomy. She has had multiple post-operative complications and has had to undergo three more operations. During the current admission she reports low mood that lifts when her family visit and frustration at being away from her husband and two children for so long. She particularly enjoys the time she spends in the hospital café with the children when they visit. Her sleep is broken but she believes it is because of abdominal pain and because she is being fed through a percutaneous endoscopic gastrostomy (PEG) tube. She is not sure about her concentration - she is able to watch the bedside TV but finds reading books an effort. She is hopeful about the future and does not have thoughts of selfharm or suicide.

Impression: Given the context of the low mood, the absence of a history of depression, her reactive mood and the lack of unequivocal biological features of depression, I consider Ms P to be experiencing an adjustment disorder with depressed mood rather than a depressive episode.

Plan

1 There is no current indication for antidepressant medications

2 Current level of risk does not require observation by a mental health nurse

3 Ms $\mathrm{P}$ is unsure about prognosis and discharge plans - could a member of the surgical team please meet with her and her husband to discuss these?

$4 \mathrm{Ms} \mathrm{P}$ is a devout Catholic and admitted to moments of feeling let down by God. I discussed a referral to the hospital chaplain and she is agreeable. Could you please refer her to the Catholic chaplain?

5 I have given her thought diaries to complete and will review these with her later this week

6 In the meantime, if there are any new psychiatric concerns please contact the psychiatry team on bleep $x x x x$

\section{Judgement of successful assessment}

\section{Basics of good practice}

Ensuring that the psychiatric assessment and intervention are successful can often be trickier than in other settings in psychiatry, as the psychiatrist is one among many clinicians seeing the patient during the hospital admission. On a basic level, it is important to talk to the medical team to ensure that their questions have been answered and that they are happy with the level of support and follow-up offered. Pasnau (1985) wrote a set of 'ten commandments' on medical etiquette for psychiatrists, which we have reproduced and interpreted in non-Biblical language in Box 5. Following these simple suggestions should satisfy most referring physicians.

\section{Measures of quality}

It can be difficult to establish clear quantitative or qualitative outcome measures. Disease-specific outcome measures are useful in certain circumstances, but not when the focus of the consultation is directed at the referrer or the ward system. Although generic outcome measures such as the

MCO answers

$1 d \quad 2 c \quad 3 b \quad 4$ e 5 e
Health of the Nation Outcome Scales (HoNOS) are becoming increasingly common in psychiatry, they are not well suited for use in the general hospital unless adapted (Ranjith 2010). In our opinion, the best approach to outcome measurement is the 'balanced score card', which combines process measures such as timeliness of response, clinicianand patient-rated outcome measures and patientand referrer-rated experience measures (Fossey 2014). The last is important, as in many general hospital consultations it can be argued that the referrer is the main 'service user' and their views are central to judging the quality of the service (Solomons 2011). The obvious advantage with this is that it creates a dialogue between services, allowing for further collaboration and education.

\section{Conclusions}

This discussion is not intended to be a comprehensive account of all issues involved in psychiatric assessments on medical wards, and it does not cover circumstances specific to the assessment of children or older adults, or specialist areas such as neuropsychiatry or perinatal psychiatry. But we hope that it addresses the basic practicalities and

BOX 5 Ten commandments on medical etiquette for psychiatrists

1 Thou shalt love thy fellow physician as thyself Talk to the referrer beforehand to clarify the reason for referral

2 Thou shalt not procrastinate Act in a timely manner

3 Thou shalt not obfuscate Avoid jargon or irrelevant psychiatric detail

4 Thou shalt be concrete Give clear and simple recommendations

5 Thou shalt honour thy patient's spouse, children and parents Don't forget the family and social situation in the plan

6 Thou shalt not hibernate Ensure that the outcome of the consultation is communicated to the referrer

7 Thou shalt persevere Return in a timely manner and ensure follow-up

8 Thou shalt not preach Do not inflict unnecessary psychiatric teaching/advice on physician colleagues

9 Thou shalt not steal thy fellow physician's patients Ensure that treatment recommendations are discussed and approved by the referrer

10 Thou shalt not shirk thy duty to thy hospital medical staff or thy local medical society Get more involved with behind-the-scenes activity at the hospital (not discussed in this article)

(Adapted from Pasnau 1985) 
will give more confidence and motivation to all psychiatrists working with patients admitted to a general hospital. The expectation of $24 / 7$ services in general hospitals cannot be delivered by liaison psychiatrists alone and we hope that our article stimulates interest in this increasingly important area of psychiatry.

\section{References}

Alexander T, Bloch S (2002) The written report in consultation-liaison psychiatry: a proposed schema. Australian and New Zealand Journal of Psychiatry, 36: 251-8.

Bazire S (2014) Psychotropic Drug Directory 2014. Arnold Rheinhold.

Department for Constitutional Affairs (2007) Mental Capacity Act 2005: Code of Practice. TSO (The Stationery Office).

Fossey M, Parsonage M (2014) Outcomes and Performance in Liaison Psychiatry. Centre for Mental Health.

Griffith JL, Gaby L (2005) Brief psychotherapy at the bedside: countering demoralization from medical illness. Psychosomatics, 46: 109-16.

House A, Akagi H (2012) Basic skills and competencies in liaison psychiatry. In Seminars in Liaison Psychiatry (2nd edn) (eds E Guthrie, S Rao, M Temple): 1-14. RCPsych Publications.

Kontos N, Freudenreich 0, Querques J, et al (2003) The consultation psychiatrist as effective physician. General Hospital Psychiatry, 25: 20-3.

Kontos N, Freudenreich 0, Querques J (2008) Ownership, responsibility and hospital care: lessons for the consultation psychiatrist. General Hospital Psychiatry, 30: 257-62

Lackamp JM (2010) The consultation process. In Psychosomatic Medicine: An Introduction to Consultation-Liason Psychiatry (eds JJ Amos, RG Robinson): 1-14. Cambridge University Press.

Lipowski ZJ (1967) Review of consultation psychiatry and psychosomatic medicine II: Clinical Aspects. Psychosomatic Medicine, 29: 201-24.

Lyketsos CG, Chisolm MS (2009) The trap of meaning: a public health tragedy. JAMA, 302: 432-3.
Ministry of Justice (2008) Mental Capacity Act 2005: Deprivation of Liberty Safeguards. Code of Practice to Supplement the Main Mental Capacity Act 2005 Code of Practice. TSO (The Stationery Office).

O'Keeffe N, Ranjith G (2007) Depression, demoralisation or adjustment disorder? Understanding emotional distress in the severely medically ill. Clinical Medicine, 7: 478-81.

Pasnau RO (1985) Ten commandments of medical etiquette for psychiatrists. Psychosomatics, 26: 128-32.

Querques J, Stern TA (2010) Approach to consultation psychiatry: assessment strategies. In Massachusetts General Hospital Handbook of General Hospital Psychiatry (6th edn) (eds JA Stern, GL Fricchione, NH Cassem, et all: 7-14. Saunders Elsevier.

Ranjith G (2010) Routine outcome measures in liaison psychiatry. The Psychiatrist, 34: 498

Ranjith G (2012) Classification and diagnosis. In Seminars in Liaison Psychiatry (2nd edn) (eds E Guthrie, S Rao, M Temple): 25-39. RCPsych Publications

Royal College of Psychiatrists (2009) Physical Health in Mental Health: Final Report of a Scoping Group (Occasional Paper OP67). Royal College of Psychiatrists

Smith GC (1998) From consultation-liaison psychiatry to psychosocial advocacy: maintaining psychiatry's scope. Australian and New Zealand Journal of Psychiatry, 32: 753-61.

Smith FA, Levenson JL, Stern TA (2011) Psychiatric assessment and consultation. In The American Psychiatric Publishing Textbook of Psychosomatic Medicine: Psychiatric Care of the Medically III (2nd edn) (ed JL Levenson): 3-18. American Psychiatric Publishing.

Solomons LC, Thachil A, Burgess C, et al (2011) Quality of psychiatric care in the general hospital: referrer perceptions of an inpatient liaison psychiatry service. General Hospital Psychiatry, 33: 260-6.

Taylor D, Paton C, Kapur S (2012) The Maudsley Prescribing Guidelines in Psychiatry (11th edn). Wiley-Blackwell.

Yager J (1989) Specific components of bedside manner in the general hospital psychiatric consultation: 12 concrete suggestions. Psychosomatics, 30: 209-12.

Zigmond (2014) A Clinician's Brief Guide to the Mental Health Act (3rd edn). RCPsych Publications.
MCOs

Select the single best option for each question stem

1 When interviewing a patient on a medical ward the psychiatrist:

a should avoid physical contact with the patient

b should not disclose that they are from the psychiatric team

c should avoid questions about the presenting medical illness

d should avoid colluding with the patient's hopelessness

e should avoid discussing the patient's views about the nurses and physicians on the ward.

2 Which of the following is likely to be accepted as an appropriate psychiatric referral from a medical ward?

a mood assessment in a 26-year-old athlete who burst into tears when told that he needed to have an above-knee amputation

b psychiatric assessment in a 45-year-old man with a 20-year history of alcohol dependence who is anxious when the daily dose of chlordiazepoxide is reduced from 80 to $60 \mathrm{mg}$ c medication review of a patient with recurrent depressive disorder who has become withdrawn 3 weeks after citalopram was stopped following gastrointestinal bleeding

$\mathrm{d}$ the partner of an intravenous drug-using patient who became aggressive when challenged about bringing drugs into the ward

e resolution of the dispute between the adult children of a 70-year-old man in the intensive care unit about turning off the ventilator.

3 According to Pasnau's adapted commandments on medical etiquette for psychiatrists in the general hospital:

a the psychiatrist should use each consultation as an opportunity to teach junior doctors the finer points of psychopathology

b psychiatrists should speak to the referrers personally about each patient seen

c the psychiatric consultation note should refer to defence mechanisms and detailed assessment of personality traits

d psychiatrists should avoid speaking to the patients' relatives and carers, for fear of breaching confidentiality e psychiatric management plans must make reference to group dynamics on the ward and countertransference reactions.

4 Which of the following is not a potential measure for judging the outcome of a psychiatric consultation on a medical ward?

a change in a patient-rated symptom scale b change in a clinician-rated symptom scale

c a patient satisfaction questionnaire

d a referrer satisfaction questionnaire

e completion of a risk assessment tool.

5 Which of the following is not a good practice point in preparing for a psychiatric assessment on a medical ward?

a talk to the referrer before you see the patient

b make sure that the patient is aware of the referral

c review the medication chart

d review all investigations carried out

e make sure that the patient is fully medically cleared before the assessment. 